\title{
Chemical extraction controlled by (FPGA), programmed by (VHDL).
}

\author{
Zuhir Nemer Alaaraj ${ }^{1}$, Abdelrasoul Jabar Alzubaidi² \\ 1 Sudan Academy of Sciences (SAS); Council of Engineering Researches \& Industrial Technologies \\ 2 Electronic Dept. - Engineering College -Sudan University of science and Technology
}

\begin{abstract}
The field programmable gate array (FPGA) is an integrated circuit designed to be configured by the customer or designer after manufacturing - hence field programmable". The FPGA configuration is generally specified using a hardware description language (HDL), similar to that used for an application-specific integrated circuit (ASIC). FPGAs can be use to implement any logical function that an ASIC could perform.

FPGAs contain programmable logic components called "logic blocks", and a hierarchy of reconfigurable interconnects that allow the blocks to be "wired together"-somewhat like many (changeable) logic gates that can be inter-wired in (many) different configurations, as shown in figure (1). Logic blocks can be configured to perform complex combinational functions, or merely simple logic gates like AND and XOR. In most FPGAs, the logic blocks also include memory elements, which may be simple flip-flops or more complete blocks of memory.
\end{abstract}

There are numerous options for designers in selecting a hardware platform for custom electronics design, ranging from embedded processors, application specific integrated circuits (ASICs), programmable microprocessor, FPGAs to programmable logic devices (PLDs). The decision to choose a specific technology such as an FPGA should depend primarily on the design requirements. Therefore, if the hardware requirements require a higher level of performance, say up several $100 \mathrm{MHz}$ operation, then an FPGA offers a suitable level of performance. [4]

To define the behavior of the FPGA is performed by programming it by a very high speed integrated circuit hardware description language (VHDL). [1]

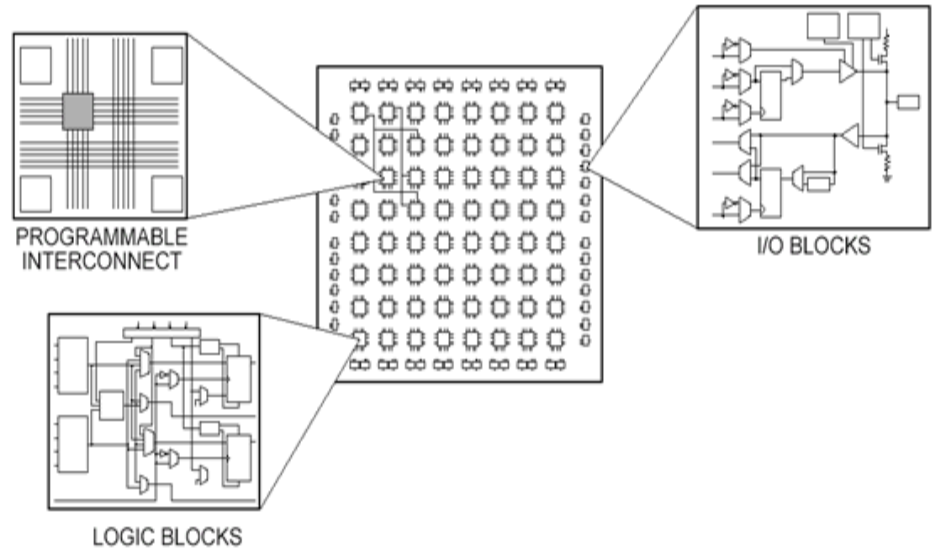

Figure (1) internal structure of FPGA

I. PROBLEM STATEMENT

It's a multi-sensing control system design based on FPGA and programmed by VHDL language .Its objective is to build a chemical extraction instrument that depends on the boiling point of the components. We took here as example of extraction of acetone from water (acetone 60\% and water 40\%).

\section{METHODOLOGY}

This paper explains the methodology of programming the FPGA device, as a control device. The following schematic diagram -figure (2) - shows the basic parts of this process. 


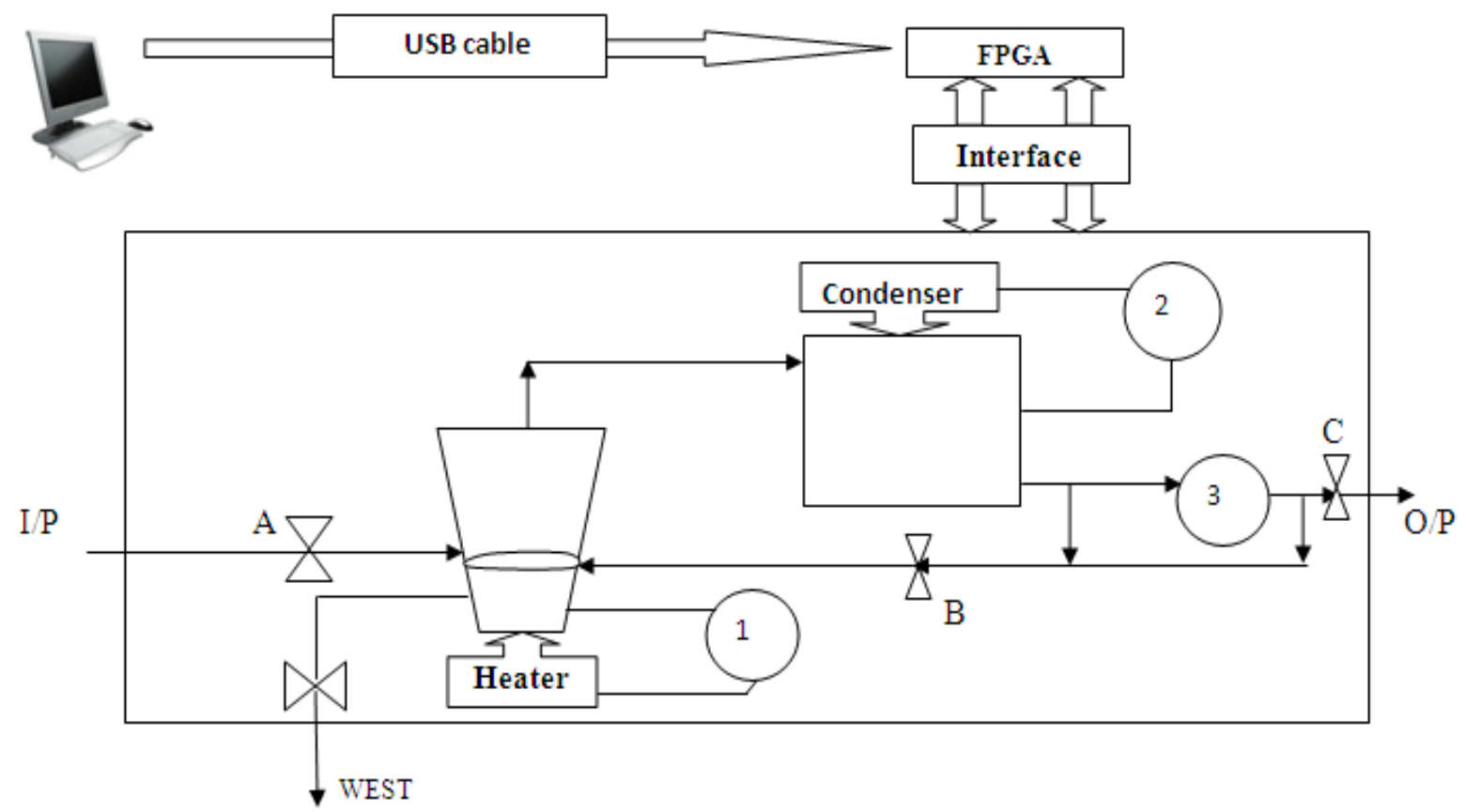

Figure (2) Basic Parts of Extraction Instrument

- A, B \&C: valves.

- 1,2 : temperature sensors.

- 3: concentration sensor.

\section{OBJECTIVES}

1. Develop a computer program - VHDL - language.

2.Download the program into FPGA.

3.Program the FPGA as multi-sensing control system of chemical compounds extraction instrument.

\section{SOFTWARE PROGRAM \& ALGORITHMS}

To achieve the objective of control, we divide the program into four parts and then compile all the four parts in top module as shown in figure (3).

The result is giving pure acetone (99\%).

The program controls the system by sensing the purity. If the output is not the demanded concentration, valve (B) opens and valve (C) closes to repeat the operation -figure (2).

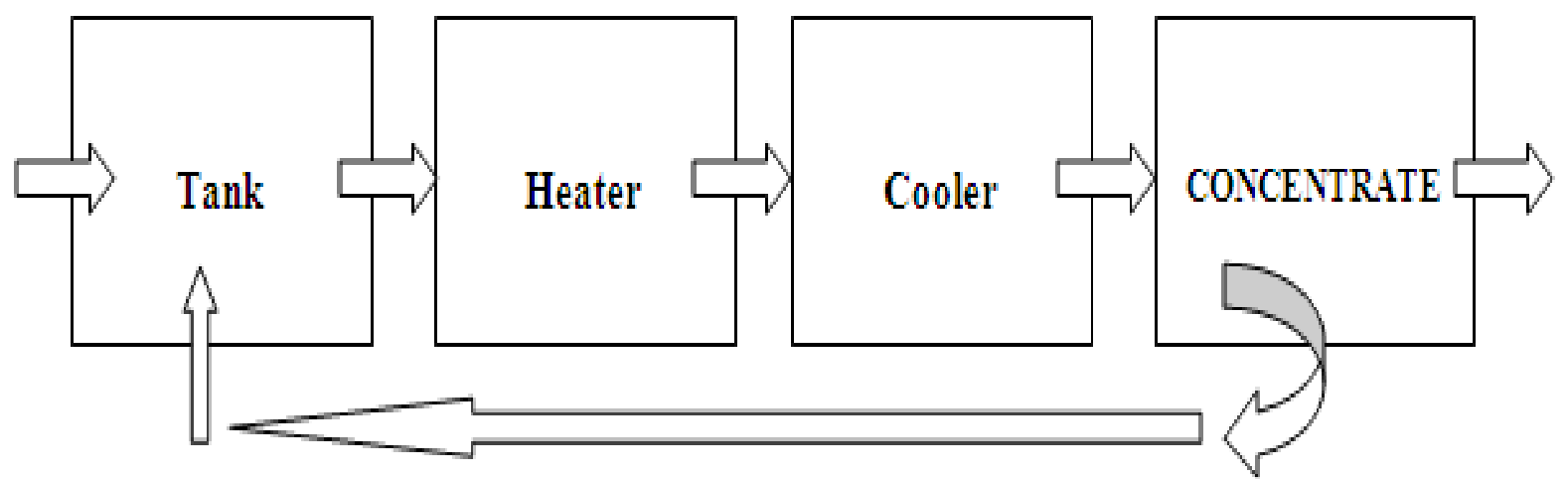

Figure (3) Four Programs Code

V.

VHDL SYNTHESIS

VHDL synthesis is the next step in the design, which converts the design in the behavioral description file into gates. The synthesis tools figure out what gates to be used based on a VHDL program file. 


\section{DESIGN IMPLEMENTATION}

* The Components for the design are:

1. General Architecture of FPGA.

2. LM 35 Temperature Sensor.

3. Valves.

4. Concentration Sensor.

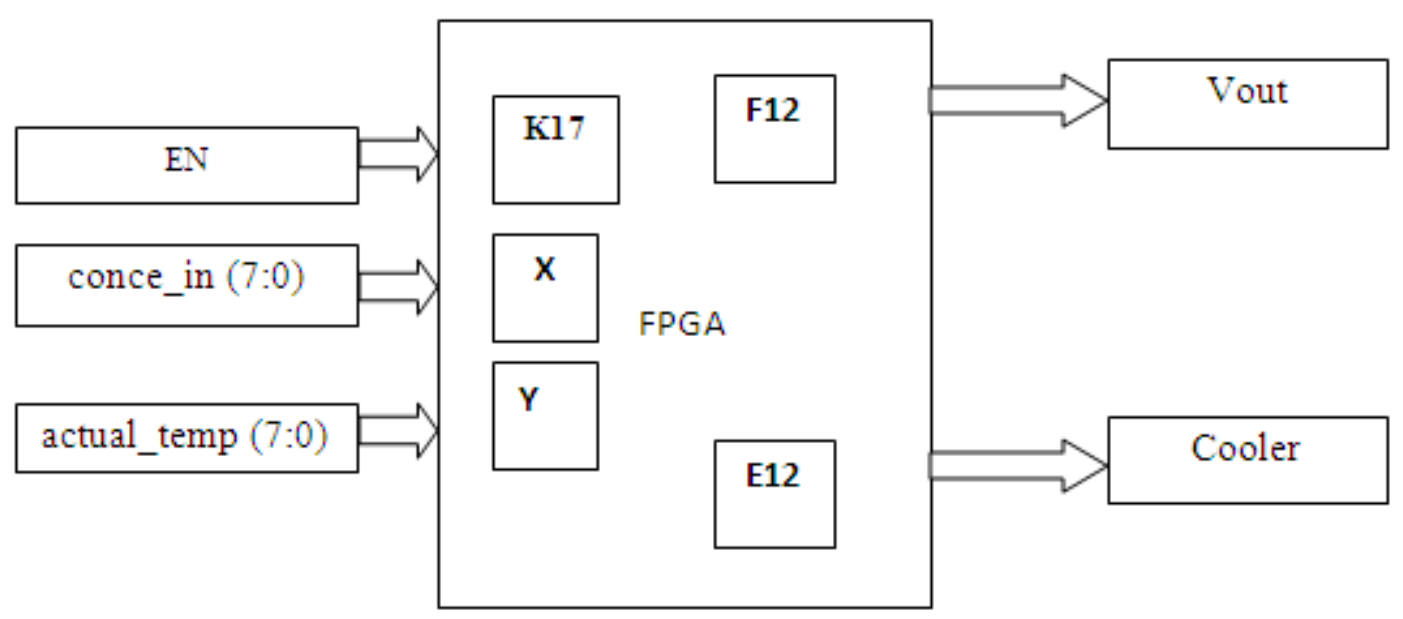

Figure (4) Block Diagram of IPs/OPs of FPGA

X: FPGA pins no. (7:0): A7, L13, L14, A8, A11, C4, H18 and N17.

Y: FPGA pins no. (7:0): D13, E13, H13, V4, D18, G9, A16 and A14.

\section{RESULT \&OBSERVATION}

The control system is programmed by VHDL language, then downloaded to the FPGA .Hence a designed control system of extraction acetone from water based on different boiling points of the compound is achieved.

We found out that FPGA has huge facilities to control systems according to the ability of the FPGA to execute the commands in a parallel way that widens the range of controlled elements with less delay time. The same thing in VHDL language, in spite of its complicated language, but we think that it is the right choice to program complicated control system. It is suitable for a system that needs fast execution of commands. [2] The disadvantage of FPGA is high cost compared to other controller devices.

The relationship between inputs and outputs are shown in tables (1) and (2).

\begin{tabular}{|l|l|l|l|l|l|l|l|l|}
\hline \multicolumn{7}{|c|}{ Input } & output \\
\hline A14 & A16 & G9 & D18 & V4 & H13 & E12 & D13 & E12 \\
\hline 0 & 1 & 1 & 1 & 0 & 0 & 0 & 0 & 1 \\
\hline \multicolumn{7}{|c|}{ Else } & 0 & 0 \\
\hline
\end{tabular}

Table (1) Output (cooler)

\begin{tabular}{|c|c|c|c|c|c|c|c|c|}
\hline \multicolumn{8}{|c|}{ Input } & \multirow{2}{*}{$\begin{array}{l}\text { output } \\
\text { vout(FPGA pin) }\end{array}$} \\
\hline \multicolumn{8}{|c|}{ Conce_in: (FPGA pins) } & \\
\hline A7 & L13 & L14 & A8 & A11 & $\mathrm{C} 4$ & H18 & N17 & F12 \\
\hline 0 & 1 & 1 & 0 & 0 & 0 & 1 & 1 & 1 \\
\hline \multicolumn{8}{|c|}{ Else } & 0 \\
\hline
\end{tabular}

Table (2) Output (Vout)

VIII.

CONCLUSION

A problem usually has multiple solutions, and a process can usually be controlled using different controllers based on different methods. Almost every control method has its merits and weaknesses. What is important is to use the right controller to fit the application at a minimum cost.

With continuous development and progress in electronics fields and control systems, the answer of this question is always fluctuant or uneven. 
In the recent years, there has been a technical revolution in the semiconductor industry and in the electronics industry, which has significantly developed the existing technologies in industrial control.

This technical development in both the semiconductor and the electronics industries have evolved industrial control into both real-time control and distributed control. Real-time control requires controllers to capture all the significant target activities and to deliver their responses as swiftly as possible so that system performance is never degraded. Distributed control indicates that controls are performed by a number of controllers and executed in a group of independent agents or units that are physically and electronically connected and communicate with each other. This tendency in industrial control has led to the future continuation of both realtime control and distributed control.

The (FPGA) have now approached intelligence similar to that of microprocessors, so that they are performing more important functional role in various control systems.

\section{REFERENCES}

[1] Volnei A. Pedroni, Circuit Design with VHDL, MIT press, Massachusts, 2004.

[2] Peng Zhang, Industrial Control Tecnology,William Andrew, N. Y., 2008.

[3] ISE Simulator (ISim), UG682 (v1.0), 2009.

[4] www.fpga4fun.com. 